\title{
Detection of the CD56+/CD45- immunophenotype by flow cytometry in neuroendocrine malignancies
}

\author{
G J Bryson, D Lear, R Williamson, R C W Wong
}

J Clin Pathol 2002;55:535-537

\begin{abstract}
Aims: Antibodies against CD56 are primarily used in flow cytometric studies to detect natural killer cells. However, they may be useful in the identification of neuroendocrine malignancies, especially if the cells do not express CD45, indicating a non-leucocyte origin.

Methods: A retrospective review was conducted on all solid tissue flow cytometric studies performed between January 1997 and September 2001, to identify all cases with a CD56+/CD45-immunophenotype.

Results: Twelve neuroendocrine malignancies (five metastatic small cell carcinomas, three Merkel cell carcinomas, two metastatic undifferentiated neuroendocrine carcinomas, one metastatic pancreatic neuroendocrine carcinoma, and one neuroblastoma) were identified.

Conclusions: CD56+/CD45- neuroendocrine malignancies are only rarely detected in the flow cytometric analysis of solid tissue samples. However, the recognition of this immunophenotype is important to avoid their misclassification as natural killer cell malignancies. Furthermore, flow cytometry assists in the rapid identification of such cases, so that appropriate immunohistochemical studies can be performed to facilitate their correct diagnosis.
\end{abstract}

C D56 is the cluster differentiation (CD) assignment for the neural cell adhesion antigen, a membrane glycoprotein widely containing immunoglobulin-like domains. ${ }^{-3}$ CD56 is of the cerebellum and cerebral cortex, and is strongly expressed throughout the grey and white matter of the spinal cord. ${ }^{1} \mathrm{NK}$ cells principally express the $140 \mathrm{kDa}$ isoform of CD56.

The use of anti-CD56 antibodies, in immunohistochemical studies, has been reported to be of value in the diagnosis of neuroendocrine $e^{4}$ (for example, neuroblastoma, Merkel cell, and small cell carcinoma) and neuroectodermal ${ }^{5}$ (for example, Ewing's sarcoma) malignancies. In contrast, anti-CD56 antibodies are primarily used in flow cytometric studies to detect NK cells, although their usefulness in the detection of neuroectodermal malignancies, ${ }^{6}$ and their binding to small cell carcinoma cell lines ${ }^{7}$ have been reported.

CD59 is expressed on a variety of cell types including natural killer cells, certain T cell subsets, large granular lymphocyte leukaemias, some myeloid leukaemias, and cells of neural origin. ${ }^{2}$

We now report a characteristic CD56+/CD45- immunophenotype in 12 neuroendocrine malignancies, with the absence of CD45 expression consistent with their non-leucocyte origin. ${ }^{8}$

\section{METHODS}

Since 1997, the immunology laboratory of Queensland Health Pathology Services at the Royal Brisbane Hospital has used

\begin{tabular}{|c|c|c|}
\hline Case & Specimen & Final diagnosis \\
\hline 1 & Lymph node & Merkel cell carcinoma \\
\hline 2 & Parotid gland & $\begin{array}{l}\text { Metastatic small cell carcinoma (unknown } \\
\text { primary) }\end{array}$ \\
\hline 3 & Lymph node & $\begin{array}{l}\text { Metastatic small cell carcinoma (unknown } \\
\text { primary) }\end{array}$ \\
\hline 4 & Lymph node & $\begin{array}{l}\text { Metastatic undifferentiated neuroendocrine } \\
\text { carcinoma }\end{array}$ \\
\hline 5 & Lymph node & $\begin{array}{l}\text { Metastatic pancreatic neuroendocrine } \\
\text { carcinoma }\end{array}$ \\
\hline 6 & Lymph node & Merkel cell carcinoma \\
\hline 7 & Lymph node & $\begin{array}{l}\text { Metastatic undifferentiated neuroendocrine } \\
\text { carcinoma }\end{array}$ \\
\hline 8 & Lymph node & Merkel cell carcinoma \\
\hline 9 & Lymph node & Metastatic small cell carcinoma (lung primary \\
\hline 10 & Lymph node & $\begin{array}{l}\text { Metastatic small cell carcinoma (unknown } \\
\text { primary) }\end{array}$ \\
\hline 11 & $\begin{array}{l}\text { Chest wall } \\
\text { mass }\end{array}$ & $\begin{array}{l}\text { Metastatic small cell carcinoma (unknown } \\
\text { primary) }\end{array}$ \\
\hline 12 & Temple mass & Neuroblastoma \\
\hline
\end{tabular}

anti-CD56 antibodies conjugated to phycoerythrin (antiCD56-PE) in the flow cytometric evaluation of tissue specimens for suspected lymphoma. Anti-CD56-PE, derived from the N901 (NKH-1) clone, ' was used in the following three colour fluorochrome combination on a Beckman Coulter XL (Beckman Coulter, Miami, Florida, USA) flow cytometer: anti-CD56-PE, anti-CD3-FITC (fluorescein isothiocyanate) and anti-CD45-PECY5 (phycoerythrin-cyanin 5 tandem). All three conjugated monoclonal antibodies were supplied by Beckman Coulter.

A retrospective review was conducted of all such studies performed between January 1997 and September 2001 to identify all cases with a CD56+/CD45- immunophenotype.

\section{RESULTS}

From approximately 2000 studies, we identified 12 specimens that expressed the CD56+/CD45- immunophenotype (table 1). All cases were neuroendocrine malignancies, comprising five metastatic small cell carcinomas (four from an unknown primary, one from a lung primary), three Merkel cell carcinomas, two metastatic undifferentiated neuroendocrine carcinomas, one metastatic pancreatic neuroendocrine carcinoma, and one neuroblastoma.

All 12 cases demonstrated the same immunophenotype, with expression of CD56 but no expression of T cell antigens (CD3, CD5, CD7), B cell antigens (CD19, CD20), or the

Abbreviations: $C D$, cluster differentiation; FITC, fluorescein isothiocyanate; NK, natural killer; PE, phycoerythrin; PECY5, phycoerythrin-cyanin 5 tandem 

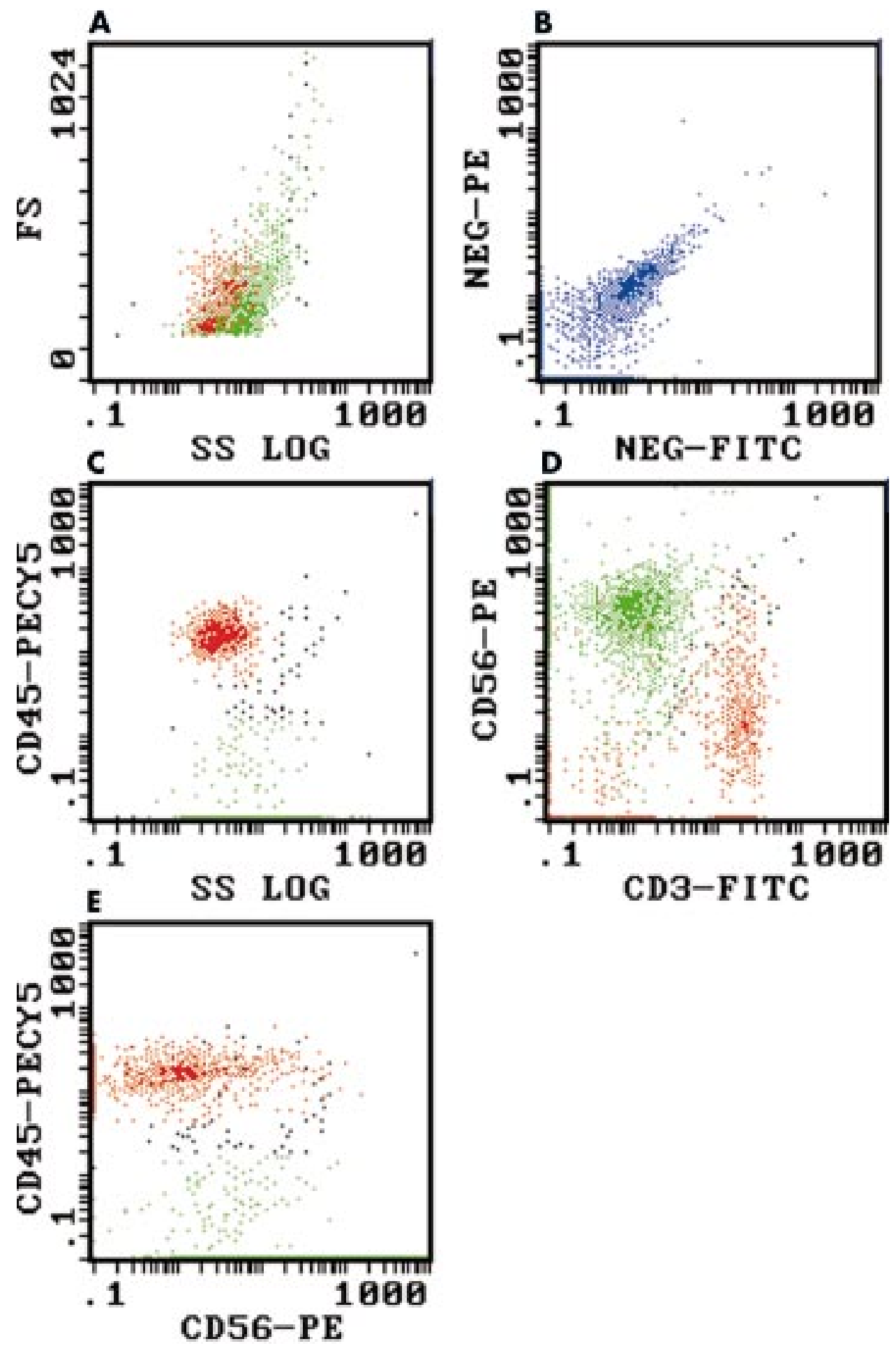

Figure 1 Flow cytometric dot plots of a metastatic small cell carcinoma of unknown primary (case 10), demonstrating CD56 expression in the absence of CD45 expression. The lymphoid (red) and small cell carcinoma populations (green) were colour backgated from the log $90^{\circ}$ side scatter versus anti-CD45-PECY5 (phycoerythrin-cyanin 5 tandem) plot (see (C) below). (A) Log $90^{\circ}$ side scatter (SS) versus forward scatter (FS): the lymphoid population (red) overlaps the small cell carcinoma population (green). (B) FITC (fluorescein isothiocyanate) versus PE (phycoerythrin) isotype controls: demonstrating the presence of significant autofluorescence/non-specific staining (blue) in this sample. (C) Log $90^{\circ}$ side scatter versus anti-CD45-PECY5: the lymphoid population (red) is clearly CD45+ whereas the small cell carcinoma population (green) is CD45-, although autofluorescence/non-specific staining results in apparent weak CD45 expression. (D) Anti-CD3-FITC versus anti-CD56-PE: the small cell carcinoma population (green) is CD56+/CD3- (autofluorescence/non-specific staining again results in apparent weak CD3 expression) and clearly distinct from the lymphocytes (red). (E) Anti-CD56-PE versus anti-CD45-PECY5: the small cell carcinoma population (green) is CD56+/CD45- (apparent weak CD45 expression as a result of autofluorescence/non-specific staining), whereas the lymphocytes (red) are CD45+.

common leucocyte antigen CD45. Figure 1 shows a representative flow cytometric study from case 10 (metastatic small cell carcinoma) demonstrating the CD56+/CD45- immunophenotype. It is important to note that significant autofluorescence/non-specific staining is often exhibited by non-haematopoietic malignancies (fig 1B). Therefore, care should be taken not to misinterpret such autofluorescence/ non-specific staining as weak CD45 expression (fig lC,E).

\section{DISCUSSION}

In our laboratory, CD56+/CD45- neuroendocrine malignancies are only rarely detected in solid tissue specimens, with 


\section{Take home messages}

- Anti-CD56 antibodies should be included in flow cytometric studies of solid tissue specimens wherever possible

- Recognition of the CD56+/CD45- immunophenotype can help avoid the potential misclassification of such specimens as natural killer cell malignancies, especially if autofluorescence/non-specific staining is misinterpreted as weak CD45 expression

- The CD56+/CD45- immunophenotype is a useful rapid indicator for the presence of neuroendocrine malignancies, thus leading to the appropriate immunohistochemical studies necessary to facilitate their correct diagnosis

only 12 cases seen in approximately 2000 specimens (0.6\%) over five and three quarter years. However, this figure is probably an underestimate, because the anti-CD56-PE/anti-CD3FITC/anti-CD45-PC5 combination was not used in studies where the number of retrieved cells was limited-for example, small biopsy specimens.

Anti-CD56 antibodies have been reported to be of value in the identification of neuroendocrine malignancies by immunohistochemical studies. ${ }^{45}$ Our cases illustrate that anti-CD56 antibodies can also be used in flow cytometric studies to identify these malignancies, with the added advantage that the concurrent use of anti-CD45 and anti-CD56 antibodies in three (or more) colour fluorochrome antibody panels also enables demonstration of the non-leucocyte lineage (negative CD45 expression) $)^{8}$ of these malignancies.

Three of the 12 CD56+/CD45- malignancies were Merkel cell carcinomas, sometimes referred to as primary neuroendocrine carcinomas of the skin. ${ }^{5}$ These uncommon malignancies exhibit the following immunohistochemical characteristics: staining for keratin 20 (approximately $90 \%$ of cases), chromogranin A (approximately 33\% of cases), CD57 (approximately $15 \%$ of cases), and synaptophysin in a minority of cases. ${ }^{5}$ Our study demonstrates the usefulness of the CD56+/CD45immunophenotype in raising the possibility of Merkel cell carcinoma, although this immunophenotype is not specific for this particular neuroendocrine malignancy.

"Our study demonstrates the usefulness of the CD56+/ CD45- immunophenotype in raising the possibility of Merkel cell carcinoma"

The detection of CD56 and CD57 expression on neuroectodermal malignancies by flow cytometry has been reported previously, ${ }^{6}$ but the expression of CD45 in these cases was not mentioned, probably because only two colour flow cytometric studies were performed. However, because neuroectodermal malignancies are not routinely sent to our department for flow cytometric studies, we were unable to determine whether these malignancies would also demonstrate the CD56+/ CD45- immunophenotype. Furthermore, because we do not routinely test for CD57 expression, we are unable to comment on whether the 12 neuroendocrine malignancies in our series would have also expressed CD57.

Our study highlights the usefulness and importance of including anti-CD56 antibodies in flow cytometric studies of solid tissue specimens wherever possible. Furthermore, the recognition of this CD56+/CD45- immunophenotype is important to avoid the potential misclassification of such specimens as NK cell malignancies, especially if autofluorescence/non-specific staining is misinterpreted as weak CD45 expression.

In conclusion, we report that flow cytometric detection of the CD56+/CD45- immunophenotype is a useful rapid indicator for the presence of neuroendocrine malignancies, thus leading to the appropriate immunohistochemical studies necessary to facilitate their correct diagnosis.

\section{Authors' affiliations}

G J Bryson, R C W Wong, Division of Immunology, Queensland Health Pathology Services, Royal Brisbane Hospital, Herston, Queensland 4029, Australia

R Williamson, Division of Anatomical Pathology, Queensland Health Pathology Services

D Lear, Sullivan Nicolaides Pathology, Taringa, Queensland 4068, Australia

Correspondence to: Dr R C W Wong, Division of Immunology, Queensland Health Pathology Services, Royal Brisbane Hospital, Herston 4029, Queensland, Australia; richard_wong@health.qld.gov.au

Accepted for publication 4 February 2002

\section{REFERENCES}

1 Lanier LL, Testi R, Bindl J, et al. Identity of Leu-19 (CD56) leukocyte differentiation antigen and neural cell adhesion molecule. J Exp Med 1989; 169:2233-38

2 Knapp W, Dorken B, Rieber P, et al. CD antigens 1989. Blood $1989 ; 74: 1448-50$.

3 Brackenbury R. Expression of neural cell adhesion molecules in normal and pathologic tissues. Ann N Y Acad Sci 1988;540:39-46.

4 Fischler DF, Bauer TW, Tubbs RR. Tissue reactivity of anti-Leu 19. Histopathology 1992;21:563-7.

5 Wick MR. Immunohistology of neuroendocrine and neuroectodermal tumors. Semin Diagn Pathol 2000;17:194-203.

6 Gardner LJ, Polski JM, Fallon R, et al. Identification of CD56 and CD57 by flow cytometry in Ewing's sarcoma or primitive neuroectodermal tumor. Virchows Arch 1998;433:35-40.

7 Koros AM, Bey EA, Conley SL, et al. Phenotypic differences among morphologically similar small-cell carcinomas detected with a panel of monoclonal antibodies and indirect immunofluorescence and flow cytometry. Int J Cancer Suppl 1994;8:127-31.

8 Shah VO, Civin Cl, Loken MR. Flow cytometric analysis of human bone marrow. IV. Differential quantitative expression of T-200 common leukocyte antigen during normal hemopoiesis. J Immunol 1988;140:1861-7

9 Cell analysis 2000: Beckman Coulter Catalog. 2000;Bulletin 9140:117. 